\title{
Esclaves maltraités ou punis dans l'Égypte romaine
}

\author{
À la mémoire de Heinz Heinen, \\ ami et modèle trop tôt disparu
}

Après une étude sur les esclaves malfaiteurs dans laquelle j'ai montré que les esclaves de l'Égypte romaine n'étaient pas des éléments très criminogènes ni même perturbateurs, il était intéressant d'envisager le cas des esclaves ayant subi des méfaits ou des maltraitances et celui des esclaves punis pour des raisons le plus souvent inconnues $\left(^{1}\right)$. Le but de cette contribution est donc de passer en revue la documentation papyrologique relative au sujet pour tenter de répondre à quelques questions parmi lesquelles : quel genre de méfaits ou de maltraitances les esclaves subissent-ils, de la part de qui, pour quelles raisons, obtiennent-ils réparation et comment, pourquoi et comment sont-ils punis ?

La rareté des documents papyrologiques relatifs à la maltraitance des esclaves par leurs maîtres ne doit pas faire conclure à l'absence de sévices envers les esclaves. Même s'il ne s'agit que d'une comparaison dont « on ignore s'il elle correspond à la situation réelle », la phrase extraite d'une pétition adressée au préfet d'Égypte dans laquelle on se plaint que les personnes libres sont « broyées, frappées, étrillées, flagellées comme des esclaves » donne une idée de ce que le

(1) J.A. Straus, « Esclaves malfaiteurs dans l'Égypte romaine », dans G. TAllet \& Chr. ZiviECoche (édd.), Le Myrte et la rose. Mélanges offerts à Françoise Dunand par ses élèves, collègues et amis = Collection CENIM, Montpellier, 2014, pp. 23-31. Je nuancerais volontiers les propos de R.S. Bagnall, Egypt in Late Antiquity (Princeton, 1993), pp. 211-212, sur le fait que les esclaves créaient beaucoup d'ennuis et même de danger à leurs maîtres et étaient source de violence pour les autres. La documention papyrologique montre que c'est vrai pour certains d'entre eux, pas pour la majorité. Les esclaves sont sans doute des éléments anxiogènes, mais ce fait apparaît dans les sources littéraires, pas papyrologiques. Je ne reviens pas sur le cas des esclaves sanctionnés à la suite d'un méfait qu'ils ont commis. Sur le sujet, voir le paragraphe «Sanctions » dans l'article cité. Ce paragraphe, fondé sur l'étude de plusieurs plaintes, doit être complété à la lumière de l'ouvrage de B. Kelly, Petitions, Litigation, and Social Control in Roman Egypt = Oxford Studies in Ancient Documents (Oxford, 2011), dont je ne disposais pas lors de sa rédaction. Il en est de même du livre de A.Z. BRYEn, Violence in Roman Egypt. A Study in Legal Interpretation (Oxford, 2013). - La législation romaine sur l'esclave objet d'actes juridiques est étudiée par M. MorABITO, Les réalités de l'esclavage d'après le Digeste (Besançon - Paris, 1981), en part. pp. 202-222.

Chronique d'Égypte XC (2015), fasc. 179 - doi: 10.1484/J.CDE.5.107572 
personnel servile est susceptible de subir $\left({ }^{2}\right)$. Les conseils donnés dans un édit du praeses de Thébaïde, Aurélios Hérodès, sur la modération dans l'usage du fouet fournissent un témoignage supplémentaire : «La soumission aux coups de fouet... est, même pour ceux de condition servile, inopportune quoique pas totalement défendue ; mais pour les hommes libres, être soumis à un tel outrage est contraire aux lois et injuste $\left(^{3}\right) »$. La différence de traitement entre libres et esclaves est bien mise en lumière par ces deux papyrus. Elle l'est aussi par un épisode des fameux Acta Alexandrinorum qui pourrait se situer en $115\left({ }^{4}\right)$. Lors de troubles à Alexandrie, soixante Alexandrins et leurs esclaves sont condamnés. Les Alexandrins sont exilés, leurs esclaves sont décapités $\left(^{5}\right)$. Elle se trouve enfin dans le vocabulaire des pétitions. Le mot ßía, qui s'applique aux objets inanimés et aux intérêts financiers ou matériels, est utilisé pour désigner le dommage causé aux esclaves tandis que le mot űßpıৎ est réservé au tort causé aux personnes libres $\left({ }^{6}\right)$. Ainsi se confirmerait l'affirmation de A.Z. Bryen selon laquelle «more salient for petitioners, it appears, is not the distinction between individuals of varying status, but rather the distinction between free and slave, which is emphasized in both individual complaints and also by local officials when they adjudicate concerning violence or discuss the penalties that can be imposed on individuals » $\left({ }^{7}\right)$. Mais cette différence de traitement indéniable ne se limite peut-être pas aux seuls esclaves : tous les humiliores pourraient être concernés $\left({ }^{8}\right)$.

Typologie des méfaits. - Le P.Oxy. VI, 903, un document daté du IV s., donne un aperçu des méfaits dont les esclaves peuvent être victimes. Il s'agit

(2) P.Wisc. I, 33 (Arsinoïte ?, 8 septembre 147). Citation d'Iza BIEŽUŃSKA-MAŁowist, L'esclavage dans l'Égypte gréco-romaine. Seconde partie. Période romaine = Archiwum filologiczne (Varsovie, 1977), p. 139 = EAD., La schiavitù nell'Egitto greco-romano = Biblioteca di storia antica (Rome, 1984), p. 276 ; Kelly, Petitions [n. 1], p. 180 ; BRYEN, Violence [n. 1], pp. 21 ; 136.

(3) P.Oxy. IX, 1186 (Thébaïde, IV ${ }^{\mathrm{e}}$ s.). - BIEŻUŃSKA-MAŁowist, L'esclavage [n. 2], p. $139=$ EAD., La schiavitù [n. 2], p. 276 ; A.Z. BRYEN, « Visibility and Violence in Petitions from Roman Egypt », GRBS 48 (2008), p. 195, n. 32 ; ID., Violence [n. 1], pp. 135-136.

(4) J. SchwARTZ, «Quelques réflexions à propos des Acta Alexandrinorum », ZPE 57 (1984), p. 132 .

(5) BGU I, 341 = C.Pap.Jud. II, 158b = H.A. Musurillo, The Acts of the Pagan Martyrs. Acta Alexandrinorum (Oxford, 1954), IX B (pp. 58-59). Voir M.-P. 2229 où l'on trouvera de la bibliographie. Y ajouter A. HARKER, Loyalty and Dissidence in Roman Egypt. The Case of the Acta Alexandrinorum (Cambridge, 2008), pp. 89 ; 192-193. — Je plaide ici pour la plausibilité des faits rapportés même si le document peut relever du « roman historique ».

(6) BRYEN, Violence [n. 1], p. 55.

(7) BRYEN, «Visibility » [n. 3], p. 188 ; ID., Violence [n. 1], pp. 135-137.

(8) E.g. Morabito, Réalités [n. 1], pp. 77 ; 226 ; R.S. Bagnall, « Official and Private Violence in Roman Egypt », BASP 26 (1989), pp. 201-216 ; C.J. FuHRMAnN, Policing the Roman Empire (Oxford, 2012), p. 80 (cite le P.Oxy. 1186 pour illustrer le traitement différencié selon le statut). 
d'un relevé d'accusations portées par une femme contre son mari : « Il enferma ses propres esclaves, les miens, mes filles adoptives, son agent et son fils sept jours entiers dans ses caves. Il insulta ses esclaves et mon esclave Zoé et les roua de coups ». L'énumération des sévices se poursuit : l'homme a battu et torturé des esclaves avant de les interroger pour savoir si sa femme a emmené des biens de la maison. Il ordonne aussi à sa femme de renvoyer une esclave. Les esclaves sont les victimes de la mésentente complète entre les époux et, peut-être, d'une dissension d'ordre religieux : en effet, l'épouse est chrétienne ce qui semble ne pas plaire à l'époux (cf. lignes 19-22, mais voir la remarque formulée par K.G. Evans dans sa n. 17) $\left({ }^{9}\right)$. Il est toutefois nécessaire d'utiliser d'autres papyrus pour établir une typologie complète des méfaits subis par les esclaves.

Le méfait le mieux attesté dans la documentation papyrologique est le vol $\left({ }^{10}\right)$. Si cette documentation dévoile l'existence de plusieurs esclaves voleurs, elle nous montre peu d'esclaves victimes de vol $\left({ }^{11}\right)$. Lors d'une altercation avec une personne pour laquelle il s'était porté caution, l'esclave Ision a perdu une tablette à écrire et soixante drachmes d'argent. On peut soupçonner son

(9) P.Oxy. VI, 903 (Oxyrhynchos, IV s.). - R.S. Bagnall, « Slavery and Society in Late Roman Egypt », dans B. HALPERn \& D.W. Hobson (édd.), Law, Politics and Society in the Ancient Mediterranean World (Sheffield, 1993), p. 235 = R.S. BAGNALL, Later Roman Egypt : Society, Religion, Economy and Administration = Collected Studies Series, 758 (Aldershot, 2003), Article I, p. 235 ; R.S. BAGNALl, Egypt in Late Antiquity (Princeton, 1993), pp. 210-211 ; D. MonTSERRAT, Sex and Society in Graeco-Roman Egypt (Londres - New York, 1996), pp. 99-100 ; A. MARCONE, «La carcerazione nell'Egitto tardoantico », dans C. BERTRAND-DAGENBACH, A. Chauvot, J.-M. Salamito \& D. Vaillancourt (édd.), Carcer II. Prison et privation de liberté dans l'Empire romain et l'Occident médiéval = Collections de l'Université Marc Bloch - Strasbourg. Études d'archéologie et d'histoire ancienne (Paris, 2004), p. 44 ; K.G. EvANS, « Domestic Violence and Women's Rights in Roman Egypt : The Case of P. Oxy. VI.903 », http://kassevans.com/EvansKPOxy903_ViolenceAgainstWomen.pdf [2002] ; BRYEN, «Visibility » [n. 2], p. 197. — Sur la violence à l'intérieur des familles et entre époux, voir BRYEN, Violence [n. 1], pp. 179-182. J. Gascou attire mon attention sur le fait que, « à la ligne 24 du papyrus, il ne s'agit pas de 'livres' (tomos), mais de chargement de blé (gomos). La femme n'a pas pu s'acquitter de ses impôts en nature, grief suprême dans une plainte ».

(10) R. Taubenschlag, The Law of Greco-Roman Egypt in the Light of the Papyri. 332 B.C.

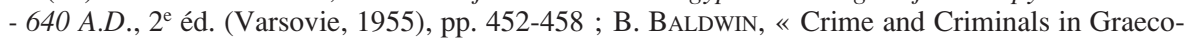
Roman Egypt », Aegyptus 43 (1963), p. 257. Selon R.W. DAVIES, « The Investigation of Some Crimes in Roman Egypt», Ancient Society 4 (1973), p. 202, le vol est un des délits les plus souvent dénoncés aux autorités militaires. Sur le vol, voir aussi H.-J. DreXHAGE, « Einbruch, Diebstahl und Strassenraub im römischen Ägypten unter besonderer Berücksichtigung der Verhältnisse in den ersten beiden Jahrhunderten n.Chr »., dans I. WEILER (éd.), Soziale Randgruppen und Aussenseiter im Altertum. Referate vom Symposion 'Soziale Randgruppen und antike Socialpolitik' in Graz (21. bis 23. September 1987) (Graz 1988), pp. 313-323 ; ID., « Eigentumsdelikte im römischen Ägypten (1.-3. Jh. n.Chr.). Ein Beitrag zur Wirtschaftsgeschichte », ANRW II, 10, 1 (Berlin - New York, 1988), pp. 952-1004 ; J.-U. KRAusE, Gefängnisse im Römischen Reich= Heidelberger Althistorische Beiträge und Epigraphische Studien 23 (Stuttgart, 1996), pp. 95-96 ; 110-111.

(11) Sur les esclaves voleurs : STRAUS, «Esclaves malfaiteurs » [n. 1], pp. 23-25. 
adversaire de les lui avoir volées $\left({ }^{12}\right)$. Épicharis, une esclave qui ne vit pas avec son maître, mais dans un appartement ou une chambre propriété d'un certain Nicanor est victime de deux vols successifs. Lors du premier cambriolage, on lui a pris des biens que la pétition ne précise pas ; lors du second, un coussin de toile, un dilasson (?) de toile, une belle tunique, une autre de toile grossière. Le papyrus est brisé ici. Le maître d'Épicharis dépose plainte auprès du stratège du nome $\left({ }^{13}\right)$. Ailleurs, des esclaves ont dépouillé leur $\sigma u ́ v \delta o v \lambda o \varsigma$, co-esclave chamelier. Leur maître les rappelle à l'ordre, leur enjoignant de restituer ce qu'ils ont volé $\left({ }^{14}\right)$. On trouve peut-être un cas supplémentaire de vol commis au détriment d'une esclave dans une lettre du $\mathrm{III}^{\mathrm{e}} \mathrm{s}$. Un certain Ptolémaios doit être entendu à propos d'une chemise de lin dont une esclave dit que « celle-ci ne lui a pas été laissée » $\left.{ }^{15}\right)$. Ptolémaios a-t-il subtilisé la chemise ?

Les violences physiques sur la personne des esclaves sont bien mieux attestées. Les esclaves sont battus ou même fouettés. Dans une procuration, le mandant autorise son représentant à emprisonner un esclave fugitif et à le battre $\left({ }^{16}\right)$. Le mari violent roue de coups ses propres esclaves et Zoé, l'esclave de son épouse $\left({ }^{17}\right)$. L'usage du fouet est évoqué dans deux documents cités plus haut : les P.Wisc. I, 33 et P.Oxy. IX, 1186. La torture avant interrogatoire est utilisée par le mari violent $\left({ }^{18}\right)$. Dans une sombre affaire de vol avec violence perpétré par un groupe d'esclaves, une bonne partie des aveux de l'un d'eux, Acholios, est obtenue sous la torture $\left({ }^{19}\right)$. Les esclaves sont victimes de coups et blessures volontaires. Ision, l'esclave de l'exégète Chairèmon, envoie lui-même une

(12) P.Ryl. II, 144 (Euhéméria, 28 mai - 24 juin 38). - BiEŻuńsKA-MaŁowist, L'esclavage [n. 2], pp. $134 ; 137$ = EAD., La schiavitù [n. 2], pp. 273 ; 275 ; Th. GRÜNEWALD, Bandits in the Roman Empire. Myth and Reality (Londres - New York, 2004), pp. 30-31 ; BRYEN, Violence [n. 1], pp. 74-79; 132, n. 18.

(13) P.Oxy. LVIII, 3916 (Oxyrhynchos, 16 février - 28 août 60). - R.S. BAGNALL, « Family and Society in Roman Oxyrhynchus », dans A.K. Bowman, R.A. Coles, N. Gonis, D. Obbink \& P.J. PARSOns, Oxyrhynchus, $a$ City and its Texts = Graeco-Roman Memoirs, 93 (Londres, 2007), p. 192. La même esclave se retrouve peut-être dans P.Oxy. II, 344 descr. édité par A. BENAISSA, « Sixteen Letters to Agoranomi from Late First Century Oxyrhynchus », ZPE 170 (2009), pp. 167-169.

(14) P.Lond. II 157a, p. 255 (II ${ }^{\mathrm{e}}$ s. ?). - BieżuńSKA-MAŁowist, L'esclavage [n. 2], p. 134, n. 103 = EAD., La schiavitù [n. 2], p. 290, n. 93.

(15) P.Hamb. IV, 255 (prov. inc., III" s.), 12-16.

(16) P.Oxy. XIV, 1643 (Oxyrhynchos, 11 mai 298). — BiEŻuńsKa-MaŁowist, L'esclavage [n. 2], pp. 139, n. 120 ; 141-142 = EAD., La schiavitù [n. 2], pp. 292, n. 120 ; 279 ; BAGNALL, " Slavery » [n. 8], p. 234 et Egypt [n. 9], p. 209 ; KRAUSE, Gefängnisse[n. 10], p. 148, n. 53. - Prisons privées en Égypte : Krause, op. cit., p. 62. M. MatTER, « Privation de liberté et lieu de détention en Égypte romaine », dans C. Bertrand-Dagenbach, A. Chauvot, M. Matter \& J.-M. Salamito (édd.), Carcer. Prison et privation de liberté dans l'Antiquité classique. Actes du colloque de Strasbourg (5 et 6 décembre 1997) = Collections de l'Université Marc Bloch-Strasbourg. Études d'archéologie et d'histoire ancienne (Paris, 1999), pp. 99-104, n'aborde pas le sujet (cf. p. 100, n. 4).

(17) P.Oxy. VI, 903. Voir n. 8.

(18) P.Oxy. VI, 903. Voir n. 8.

(19) P.Lips. I, 40 (Hermoupolis, avant 381 ?). — BRYEN, « Visibility » [n. 3], p. 191. 
pétition à l'épistate des phylacites. Il s'est porté garant pour Onnophris, fils de Silbon. Lors d'une rencontre avec celui-ci, il aborde le sujet. Onnophris le prend mal et attaque Ision. Celui-ci réclame un châtiment adéquat pour Onnophris $\left({ }^{20}\right)$. Dans la pétition suivante, le plaignant et une esclave de son fils reçoivent coups et blessures. L'esclave est blessée à la lèvre. Le plaignant demande réparation légale et traitement médical pour l'esclave $\left({ }^{21}\right)$. Dans une plainte adressée au décadarque, Héraïs dénonce des faits bien plus graves. Lors d'un vol nocturne perpétré par un ou des inconnus, trois personnes — dont Isarion, l'esclave de sa fille — sont tuées ${ }^{22}$ ). Ptolémaios fils de Ptolémaios dépose plainte contre Pétésouchos et son épouse qui ont brûlé l'esclave Harpa[ ] au point d'entraîner sa mort $\left({ }^{23}\right)$. Enfin, si l'on doit croire D. Montserrat, la mention de nombreuses cicatrices dans la description de l'esclave Procopton alias Aptos « evokes a man's body after a lifetime of hard work and probably hard knocks $\left({ }^{24}\right) »$. Mais il ne s'agit-là que d'une supposition. La violence sexuelle exercée sur les esclaves n'est guère attestée dans la documentation papyrologique $\left({ }^{25}\right)$. Pourtant le viol de l'esclave par son maître existait à coup sûr, mais je n'en trouve pas de trace. Un cas de violences sexuelles est peut-être rapporté dans un document. Une veuve porte plainte parce que les anciens du village ont saisi son esclave Sarapias, ôté les vêtements qu'elle portait et se sont livrés à des voies de fait sur elle. Leur motivation : récupérer un impôt impayé $\left({ }^{26}\right)$. Un papyrus illustre une possible relation homosexuelle forcée entre deux libres et un Epaphroditos qui, étant donné son nom, pourrait être un affranchi : «Apion et Epimas disent à leur très cher Epaphroditos : "Si tu nous laisse t'enculer cela ira bien pour toi, nous cesserons de te rosser. Si tu nous laisses t'enculer, porte-toi bien" $\left({ }^{27}\right)$ ».

(20) P.Ryl. II, 144 (Euhéméria, 28 mai - 24 juin 38). Voir n. 12.

(21) P.Oxy. XXXIII, 2672 (Oxyrhynchos, 28 septembre - 27 octobre 218). — BIEŻUŃSKAMAŁOWIST, L'esclavage [n. 2], pp. 138 = EAD., La schiavitù [n. 2], p. 276 ; BRYEN, « Visibility » [n. 3], pp. 192-193 ; ID., Violence [n. 1], pp. 106 ; 132, n. 17.

(22) P.Hamb. I, 10 (Théadelphie, II ${ }^{\mathrm{e}}$ s.), 1-12. - R.W. DAVIES, « The Investigation of Some Crimes in Roman Egypt », AncSoc 4 (1973), p. 203.

(23) SB XX, 14085 (Arsinoïte, après 29 mai 12 av. ou 32 de n.è.), col. II, 11.

(24) P.Oxy. XLII, 3054 (Oxyrhynchos, 26 mai - 24 juin 265 ?). - Montserrat, Sex and Society [n. 8], p. 57.

(25) Selon Kelly, Petitions [n. 1], p. 165, la violence sexuelle n'est attestée ni dans les pétitions ni dans les procès-verbaux d'audiences judiciaires. Elle est « under-reported » selon BRYEN, Violence [n. 1], p. 113. On ne la voit guère dans le Digeste sauf s'il s'agit de la prostitution de l'esclave par son maître, voir MoRABito, Réalités [n. 1], p. 191.

(26) SB XIV, 11904 (Tebtynis, 184). - Kelly, Petitions [n. 1], pp. 61 ; 236-237. - En Égypte, le non paiement des impôts et des amendes ainsi que la fraude fiscale constituent un crime très grave. Voir A. MARCONE, «La privation de liberté dans l'Égypte gréco-romaine », dans C. Bertrand-Dagenbach et al. (édd.), Carcer [n. 16], pp. 89-98 et la remarque de J. Gascou reprise dans la n. 9 ci-dessus.

(27) P.Oxy. XLII, 3070 (Oxyrhynchos, I ${ }^{\mathrm{er}}$ s.). - MonTSERrat, Sex and Society [n. 9], pp. 136138 ; ID., « Experiencing the male body in Roman Egypt », dans L. Foxhall \& J. SALmON (édd.), 
Parfois, les blessures sont accidentelles $\left({ }^{28}\right)$. Thermouthion, fille de Ploutarchos, porte plainte, car son esclave Peina a été renversée par un âne conduit par Polydeukès, un esclave lui aussi. Peina a le bras brisé, de très nombreux endroits du corps blessés et a perdu l'usage de la parole $\left({ }^{29}\right)$. En avril 96, Théon fils de Harpocration, médecin de la cité d'Oxyrhynchos, envoie au stratège du nome un rapport que celui-ci lui a demandé. Il était chargé d'examiner Alexandra, l'esclave de Claudia Dionysia, blessée dans des circonstances qui nous sont inconnues, mais qui requièrent l'intervention de l'autorité publique. Plaie au majeur, hématome à la poitrine, vomissements, fièvre, l'esclave est sérieusement touchée $\left({ }^{30}\right)$. De l'esclave Aieus, on ne sait s'il a été victime d'une agression ou d'un accident. Toujours est-il que son maître, le palatinus Fl. Martyrios, a demandé une expertise médicale qui est faite par quatre médecins publics mandatés par le logistès de l'Oxyrhynchite, Fl. Sarmatès $\left({ }^{31}\right)$.

Proches de la violence physique, l'enlèvement et la séquestration. Trois frères possèdent en indivision une esclave nommée Martilla. Ils vivent à Antinooupolis alors que l'esclave se trouve dans l'Arsinoïte. Deux des frères, Lysimachos alias Didymos et Philosarapis, envoient une pétition à l'épistratège : " Maintenant, un certain Sarapammon, fils de Pyrrhion, et son frère Dios, de l'Arsinoïte, résidant dans le village de Tebtynis de la méride de Polémon, l'ont saisie tandis qu'elle était au travail, l'ont enlevée par la force, sans intervention du stratège, l'ont gardée avec eux et la gardent jusqu'à présent sous le prétexte d'une créance qu'ils prétendent avoir à l'égard de notre autre frère Philantinoos alias Neilammon $\left({ }^{32}\right)$ ». Que la dette du frère soit attestée ou non, on voit ici une esclave enlevée par la force parce que les créanciers estiment pouvoir s'en servir comme moyen de remboursement. Il en est peut-être de même lorsqu'une «faible veuve » se plaint : «car Thonis, le curateur de Seuthès, s'est précipité dans ma maison et a osé enlever mon esclave Théodora quoiqu'il n'eût aucun pouvoir

When men were men. Masculinity, power and identity in classical antiquity (Londres - New York, 1998), pp. 154-157. — Sur les relations sexuelles entre maîtres et esclaves, cf. J.A. STRAUS, « L'esclavage dans l'Égypte romaine », ANRW, II, 10, 1 (Berlin - New York, 1988), p. 897 ; MonTSERRAT, Sex and Society [n. 9], pp. 101-103.

(28) Des paragraphes du Digeste nous apprennent que « l'accident de la circulation ne semble pas être chose rare », Morabito, Réalités [n. 1], pp. 184-185.

(29) P.Oxy. L, 3555 (BL X 154) (Oxyrhynchos, $\mathrm{I}^{\mathrm{er}}-\mathrm{II}^{\mathrm{e}}$ s.). - Kelly, Petitions [n. 1], pp. 236$237 ; 249$.

(30) P.Oslo III, 95 = C.Pap.Hengstl 37 (Oxyrhynchos). - M.-P. ${ }^{3} 8345$ où l'on trouvera la bibliographie. Y ajouter BRYEN, « Visibility » [n. 3], p. 197.

(31) P.Oxy. LXIII, 4370 (Oxyrhynchos, 17 novembre 354). - M.-P. ${ }^{3} 8363$ où l'on trouvera la bibliographie.

(32) P.Fam.Tebt. 37 (Antinooupolis, 4 ou 16 mars 167), 9-15. - BieżuńsKA-MaŁowist, L'esclavage [n. 2], pp. 129 = EAD., La schiavitù [n. 2], p. 268. Sur la contrainte par corps, B. AnAGNOSTOUCANAS, Juge et sentence dans l'Égypte romaine = Études de Philosophie et d'Histoire du Droit, 6 (Paris, 1981), pp. 231-234 ; MATTER, « Privation » [n. 16], p. 103. 
sur elle $\left({ }^{33}\right)$ ». Un dernier cas de séquestration est attesté : l'époux violent dont il est question en début de paragraphe a enfermé les esclaves de son épouse dans des caves pendant une durée de sept jours $\left({ }^{34}\right)$. Dans deux papyrus, des maîtres se plaignent que leurs esclaves ont été enlevés. Mais ces derniers semblent avoir mis beaucoup de complaisance à se laisser faire. Dans la première plainte, il apparaît qu'un certain Harpagathès a convaincu des esclaves de voler quelques objets à leur maître, de quitter ce dernier et de le rejoindre, lui $\left({ }^{35}\right)$. Dans la seconde plainte, on voit Hermès qui entraîne Eutychios, l'esclave d'Aurélios Didymos, à casser la porte de la maison de son maître et à lui voler des biens meubles. Didymos retrouve l'esclave chez Hermès et précise dans sa plainte qu'Eutychios «a été détourné de moi déjà souvent par Hermès $\left({ }^{36}\right)$ ». B. Kelly cite quelques papyrus dans lesquels « the person who suffered the wrong was a slave $\left({ }^{37}\right) »$. Parmi ces papyrus figurent P.Münch. III, 74 (Arsinoïte, 31 août 158) et PSI X, 1102 (Oxyrhynchos ?, c. 271-272). Dans les deux cas, il s'agit d'esclaves qui font partie d'un héritage détourné par des proches des héritiers, mineurs au moment de la mort de leur père. Sauf à considérer qu'ils sont victimes d'une sorte d'enlèvement, je ne voyais pas le tort qu'ils subissaient. B. Kelly a aimablement précisé sa pensée dans un message électronique : «My point would be that there could well have been a certain amount of physical mistreatment of the slaves (or threats thereof) involved in this ». Dans ce cas, on ajoutera aux deux papyrus qu'il cite les documents suivants attestant l'existence d'autres esclaves inclus dans des biens détournés. Dans le P.Meyer 8 (Arsinoïte, 16 août 151), des esclaves donnés en héritage à des mineurs par leur mère sont détournés par le père. Et le P.Cair.Isid. 64 (Caranis, c. 298) est une plainte dans laquelle deux esclaves font partie d'un héritage détourné par l'oncle des héritières $\left({ }^{38}\right)$. Ailleurs, une certaine Thatrès se saisit des biens de deux mineurs parmi lesquels figurent des esclaves sous le prétexte qu'elle est la sœur de leur grand-père $\left({ }^{39}\right)$.

La violence verbale n'est pas rare dans la documentation papyrologique, mais, pour ce qui est de la violence verbale envers les esclaves, un seul cas est

(33) P.Oxy. VIII, 1120 (Oxyrhynchos, déb. III s.), 13-19. - B. BALdwin, « Crime and Criminals in Graeco-Roman Egypt », Aegyptus 43 (1963), p. 261 ; Kelly, Petitions [n. 1], pp. 236237 ; BRYEN, « Visibility » [n. 3], p. 192, n. 29 ; ID., Violence [n. 1], pp. 133-134 ; 299, n. 9.

(34) P.Oxy. VI, 903 (Oxyrhynchos, IV ${ }^{\mathrm{e}}$ s.). Voir n. 9. Il y a aussi enfermé son propre fils et un intendant !

(35) $S B$ XXIV, 16257, col. I (Socnopaiou Nèsos, après le 18 octobre 123).

(36) SB XVIII, 14056 (Hermoupolis, 19 novembre 326). — BAGNALL, « Slavery » [n. 9], p. 235 ; ID., Egypt in Late Antiquity [n. 9], p. 210.

(37) Kelly, Petitions [n. 1], p. 214, n. 24.

(38) Sur les circonstances, voir BRYEN, Violence [n. 1], pp. 182-191.

(39) $B G U$ I, 168 = M.Chrest. 121 (Arsinoïte, c. 171). — Kelly, Petitions [n. 1], pp. 110-111 (corriger « claiming to be their sister » en « claiming to be the sister of their grandfather ». 
connu. Il est une fois encore le fait de l'époux violent du P. Oxy. 903 qui insulte Zoé l'esclave de sa femme $\left({ }^{40}\right)$.

Dans l'épisode des Acta Alexandrinorum cité ci-dessus, les esclaves sont victimes du comportement de leurs maîtres. Pris dans la tourmente des événements suscités par les antagonismes entre Juifs et Alexandrins, les esclaves d'Alexandrins condamnés à l'exil sont décapités. D'autres, qui espèrent trouver la sécurité auprès de leurs maîtres, sont traduits en justice par ceux-ci et subissent un châtiment. On subodore un acte de lâcheté des maîtres $\left({ }^{41}\right)$.

La fuite des esclaves peut être la conséquence d'une maltraitance. Mais aucun document relatif aux esclaves fugitifs ne donne la raison de leur fuite. Dans une déclaration censitaire de 189, cinq esclaves sont déclarées par leur maîtresse. Trois sont en fuite. Les deux qui ne se sont pas enfuies ont sans doute été retenues par la jeunesse de leurs enfants, l'aîné étant âgé de huit ans. On ne peut s'empêcher de penser que le comportement de la maîtresse est la cause de la fuite $\left({ }^{42}\right)$.

Un dernier cas ne concerne pas à proprement parler la maltraitance d'un esclave, mais l'abus de confiance. Dans le BGU IV, 1079 (Philadelphie, 4 août 41), je comprends en effet que l'esclave (ou l'affranchi) Héracleidès s'est laissé duper dans une transaction avec un certain Ptollarion et que son maître (patron) lui glisse à l'oreille le moyen de rétablir la situation : "Suis Ptollarion tout le temps. Peut-être pourra-t-il te tirer d'affaire. Dis-lui : "Moi, c'est moi, les autres, c'est les autres. Moi, je suis un esclave. Je t'ai vendu mes biens pour un talent (= trop peu ?). Je ne sais pas ce que le patron me fera. Nous avons beaucoup de créanciers. Ne nous mets pas en faillite." Demande-lui chaque jour. Peut-être prendra-t-il pitié de toi » $\left({ }^{43}\right)$. Mais, comme dans la plupart des lettres privées, les circonstances dans lesquelles ces paroles s'inscrivent restent bien obscures.

Demande de réparation et application de sanctions. - Lorsqu'un esclave subit un délit, une plainte peut être introduite dans laquelle on demande réparation du dommage subi et application de sanctions $\left({ }^{44}\right)$. Selon D. Hobson, la

(40) P.Oxy. VI, 903 (Oxyrhynchos, IV s.). Voir n. 9. - Violence verbale dans l'Égypte romaine : BRYEN, Violence [n. 1], pp. 105-112. À Rome, l'action prétorienne doit être refusée si l'esclave a essuyé une injure verbale sans importance, voir MoRABITO, Réalités [n. 1], p. 219.

(41) $B G U$ I, $341=$ C.Pap.Jud. II, 158b = H.A. MusuriLlo, The Acts of the Pagan Martyrs [n. 5], IX B (pp. 58-59). Voir n. 5.

(42) P.Berl.Leihg. I, 15 (Tebtynis, 22 août 189). — BIEŻuńSKA-MAŁowist, L'esclavage [n. 2], p. 114 = EAD., La schiavitù [n. 2], p. 257.

(43) $B G U$ IV, 1079 = W.Chrest. 60 = Sel.Pap. I, $107=$ C.Pap.Jud. II, 152 (Philadelphie, 4 août 41).

(44) Sur les plaintes dans l'Égypte romaine, voir par exemple GRÜNEWALD, Bandits [n. 12], pp. 25-31 ; Kelly, Petitions [n. 1] ; Bryen, Violence [n. 1]. La diplomatique, le vocabulaire, la 
demande de sanctions se ferait dans une moindre mesure que celle de réparation $\left({ }^{45}\right)$. Cette constatation s'appliquerait très bien au maître d'un esclave désireux d'obtenir la réparation matérielle des dégâts causés à son bien. En cas de blessure de son esclave, par exemple, le maître tente de se faire rembourser les frais de médecin. Mais B. Kelly et A.Z. Bryen pensent que la demande de sanction est présente dans beaucoup de cas $\left({ }^{46}\right)$. Dans la pétition qu'il envoie à l'épistate des phylacites, l'esclave Ision ne demande pas une réparation, mais un châtiment $\left({ }^{47}\right)$. Parfois, on réclame la poursuite du coupable sans autre précision $\left({ }^{48}\right)$. Ailleurs, on demande réparation et traitement médical $\left({ }^{49}\right)$. L'esclave n'ayant en principe pas le droit de déposer plainte, un libre s'en charge. Il s'agit le plus souvent de son propriétaire, homme ou femme, mais dans un cas, c'est la mère de la propriétaire qui agit et dans un autre l'esclave lui-même. La démarche personnelle de l'esclave Ision s'explique peut-être par la fonction importante de son maître qui est exégète $\left({ }^{50}\right)$. On relève toutefois que, selon A.Z. Bryen, la plainte d'Ision a été rejetée. En effet, le document ne comporte pas la souscription de son destinataire, l'épistate des phylacites, et n'a pas été envoyée à l'archéphode avec l'instruction que celui-ci arrête Onnophris, le contrevenant, et commence donc la procédure légale. Les autorités locales auraient estimé qu'Onnophris n'avait pas utilisé une violence inacceptable, mais une force légitime et qu'il l'avait employée contre un esclave qui la méritait. Il ne reste plus à Chairémon, le maître de l'esclave, qu'à poursuivre lui-même Onnophris pour dommage causé à son bien ${ }^{(51)}$. Dans une liste d'affaires judiciaires, le maître d'un esclave dépose plainte contre deux personnes qui ont brûlé son esclave à mort. Il souhaite sans doute obtenir la punition des coupables et une réparation pour la perte de l'esclave $\left({ }^{52}\right)$. À la suite d'une plainte

syntaxe, la manière de rapporter les faits, les requêtes des plaintes dans lesquelles un esclave est la victime ne diffèrent en rien des plaintes dans lesquelles un libre est la victime.

(45) D.W. Hobson, « The Impact of Law on Village Life in Roman Egypt », dans B. HALPERN \& D.W. Hobson (édd.), Law, Politics and Society [n. 9], pp. 193-219.

(46) Kelly, Petitions [n. 1] ; Bryen, Violence [n. 1], pp. 128-140. Voir par exemple Bryen, p. 131 : «In case where violence is involved along with damage to property, petitioners often are clear that they wish for both compensation and punishment ». La demande de réparation du dommage ou de sanctions manque à plusieurs reprises, car le papyrus est brisé à l'endroit où elle devrait figurer : e.g. P.Hamb. I, 10 ; P.Oxy. L, 3555 ; P.Oxy. LVIII, 3916.

(47) P.Ryl. II, 144 (Euhéméria, 28 mai - 24 juin 38). Voir n. 12.

(48) P.Fam.Tebt. 37 (Antinooupolis, 4 ou 16 mars 167). Voir n. 32. SB XIV, 11904 (Tebtynis, 184). Voir n. 26.

(49) P.Oxy. XXXIII, 2672 (Oxyrhynchos, 28 septembre - 27 octobre 218). Voir n. 21.

(50) Hommes : P.Oxy. LVIII, 3916 ; P.Fam.Tebt. 37 ; P. Oxy. XXXIII, 2672. - Femmes : P.Oxy. L, 3555 (BL X, 154) ; SB XIV, 11904 ; P.Oxy. 1120. — Mère : P.Hamb. I, 10. — Esclave : P.Ryl. II, 144. - Femmes et esclaves dans ce genre de requête : GrüNEWALD, Bandits [n. 12], pp. 30-31. - Femmes : Kelly, Petitions [n. 1], pp. 229-241.

(51) BRYEN, Violence [n. 1], p. 76.

(52) SB XX, 14085 (Arsinoïte, après 29 mai 12 av. ou 32 de n.è.), col. II, 11. 
déposée par le palatinus Flauius Martyrius, le logistès Flauius Sarmates ordonne à quatre médecins publics d'examiner les blessures de l'esclave Aeus. Il est évident que la démarche a pour but la réparation du dommage causé à l'esclave et à son maître et une éventuelle punition du coupable $\left({ }^{53}\right)$. On peut supposer un comportement semblable quand un médecin public d'Oxyrhynchos envoie au stratège du nome un rapport relatif à des blessures reçues par Alexandra, l'esclave de Claudia Dionysia $\left({ }^{54}\right)$. On ignore la suite donnée à toutes ces requêtes. Mais il est indéniable qu'elles ont pour but la sauvegarde des intérêts du propriétaire de l'esclave avant la réparation des torts faits à l'esclave lui-même.

Aucune protection contre la violence ? - On se souvient de l'épisode dans lequel Auguste empêche Védius Pollion de jeter aux murènes un esclave maladroit $\left({ }^{55}\right)$. Sans doute usait-il dans ce cas d'un pouvoir discrétionnaire, celui de l'empereur tout puissant. Mais il n'est pas exclu qu'il ait agi en vertu de son pouvoir censorial. En effet, à Rome, le censeur avait parmi ses attributions la tâche de surveiller les agissements des maîtres des esclaves. À l'époque impériale existe une législation susceptible de protéger les esclaves contre les méfaits commis par leurs maîtres ou d'autres libres. Mais son efficacité est mise en doute $\left({ }^{56}\right)$.

Qu'en est-il en Égypte ? Comme partout ailleurs dans l'antiquité, l'esclave y est considéré comme une chose. Le fait apparaît très clairement dans la documentation papyrologique : des esclaves sont vendus, légués, donnés en dot ou en gage, partagés au point d'en arriver à l'indivision. Dans certaines déclarations de recensement, il est évident que les esclaves font partie des biens immeubles. Pour cette raison, tout changement de propriétaire est enregistré à la

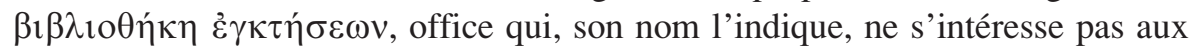
personnes mais aux biens. L'esclave est donc légalement un objet sur lequel le

(53) P.Oxy. LXIII, 4370 (Oxyrhynchos, 17 novembre 354). Voir n. 31.

(54) P.Oslo III, 95 = C.Pap.Hengstl 37 (Oxyrhynchos). Voir n. 30.

(55) Voir, par ex., SÉnÈQue, De la colère, III, 40, 2-5 ou Dion CASsius, LIV, 23, 2-5.

(56) Sur cette législation, voir, par ex., A. Piganiol, « Les empereurs parlent aux esclaves », Romanitas, 1 (1958), pp. 7-18 = ID., Scripta varia. III. L'empire = Collection Latomus, 133 (Bruxelles, 1973), pp. 202-211 ; O. RoBledA, Il diritto degli schiavi nell'antica Roma (Rome, 1976), pp. 83-88 ; Morabito, Réalités [n. 1], passim ; A. Watson, Roman Slave Law (Baltimore - Londres, 1987), pp. 115-133. - Doute sur l'efficacité : Morabito, op. cit., p. 183 ; Watson, op. cit., pp. 127-128 ; R. GAMAUF, «Sklavenschutz und römische Jurisprudenz », Orbis iuris Romani, 4 (1998), pp. 18-44 ; H. HEINEN, « Amtsärzliche Untersuchung eines toten Sklaven. Überlegungen zu P.Oxy. III 475 », dans A. MArcone (éd.), Medicina e società nel mondo antico. Atti del convegno di Udine (4-5 ottobre 2005) = Studi Udinesi sul Mondo Antico, 4 (Florence, 2006), pp. 194202. - Même la législation d'un empereur « humaniste » comme Marc Aurèle est peu favorable aux esclaves. Voir Th. FInKEnAUER, Die Rechtsetzung Mark Aurels zur Sklaverei (Mayence Stuttgart, 2010). 
maître a tous les pouvoirs même celui de l'endommager (blessures) ou de le briser définitivement (mort).

Le papyrus suivant, provenant d'Oxyrhynchos et daté de 182, pourrait suggérer que des limites sont mises à l'arbitraire des propriétaires d'esclaves. Voici les faits. Durant une fête qui avait lieu dans le village oxyrhynchite de Sénepta, l'esclave Epaphroditos est mort à la suite d'un accident. Le beau-père de son propriétaire, Léonidès alias Sérénos, introduit une requête écrite au stratège du nome pour que celui-ci envoie un de ses assistants pour mener une enquête. Le stratège nomme un assistant et lui ordonne d'aller à Sénepta, accompagné d'un médecin public, pour examiner le cadavre, délivrer un permis d'inhumer et dresser un rapport. Une telle démarche peut répondre à une triple nécessité. D'abord, respecter les obligations relatives au contrôle de la propriété et de l'aliénation des esclaves. Epaphroditos ne peut être enterré sans une autorisation afin d'éviter toute évasion fiscale. Cette explication tout à fait plausible n'est cependant pas confirmée par la documentation papyrologique connue à ce jour. Ensuite, préciser les circonstances de la mort afin de s'assurer qu'elle est accidentelle. Dans le cas contraire, il serait nécessaire de trouver le responsable de la mort qui devrait alors dédommager le propriétaire de l'esclave. Enfin, on pourrait rapprocher ce papyrus d'une constitution d'Antonin (138-161) prévoyant des sanctions contre celui qui tue son esclave sans raison : «car, en vertu d'une constitution du divin Antonin le Pieux, celui qui tue son esclave sans raison ne doit pas être moins puni que celui qui tue l'esclave d'un autre » (Justinien, Institutes 1, 8, 2. Aussi Gaius 1, 53). Dans ce cas, l'enquête porterait sur les circonstances de la mort d'Epaphroditos afin de voir s'il n'y avait pas homicide volontaire. Une plainte aurait été déposée par Léonidès alias Sérénos parce qu’il soupçonnait son beau-fils ou une autre personne d'avoir tué Epaphroditos. Ce papyrus serait donc l'illustration d'une certaine restriction au pouvoir du maître qui pourrait être puni si le médecin concluait au meurtre de l'esclave $\left({ }^{57}\right)$.

H. Heinen s'oppose avec force à cette dernière interprétation du papyrus. Selon lui, il n'est pas légitime d'établir « une relation directe entre la pratique administrative de l'Égypte romaine relative à l'examen par un médecin public du jeune esclave décédé et les dispositions d'Antonin le Pieux relatives à la protection des esclaves ». La procédure mise en pratique dans le papyrus oxyrhynchite devait certainement fixer si l'esclave avait trouvé la mort

(57) P.Oxy. III, 475 = W.Chrest. 494 = Sel.Pap. II, $337=$ C.Pap.Hengstl $95=$ G. TeDESCHI, « Lo spettacolo in età ellenistica e tardo antica nella documentazione epigrafica e papiracea », Pap. Lup., 11 (2002), pp. 173-175 (et 132-133 pour le commentaire) (Oxyrhynchos, 182). Récemment : A. RiCCIARDETTO, "'Inspecter un corps mort' : contribution des rapports médicaux sur papyrus ( $\mathrm{I}^{\mathrm{er}}-\mathrm{IV}^{\mathrm{e}}$ siècles de notre ère) à l'histoire de la pratique médico-légale antique », dans $\mathrm{Ph}$. Charlier \& D. Gourevitch (édd.), $4^{e}$ colloque international de pathographie. Saint Jean de Cole, Mai 2011 = Collection Pathographie, 9 (Paris, 2013), pp. 105-106. 
accidentellement ou non. Mais il est assez peu probable que la raison première d'un tel contrôle ait eu pour but de protéger l'esclave contre un acte de violence de son propre maître et de limiter la patria potestas dans le sens de la constitution d'Antonin le Pieux. Pour H. Heinen, l'examen obligatoire par un médecin public des cas de mort violente ou douteuse relevait du maintien de l'ordre et de la lutte contre la violence. Si des esclaves étaient morts de manière peu claire, l'enquête était motivée par des considérations fiscales. On pouvait aussi rechercher la responsabilité d'une tierce personne dans la mort de l'esclave afin de défendre les intérêts de son propriétaire. En aucun cas, il n'est question de protéger l'esclave $\left({ }^{58}\right)$.

Je suis disposé à accepter l'essentiel des conclusions auxquelles arrive H. Heinen. Mais il existe bel et bien une législation impériale susceptible de protéger peu ou prou l'esclave contre les abus de son maître. Plusieurs savants estiment que la collusion entre les maîtres d'esclaves et les représentants de la justice, eux-mêmes propriétaires d'esclaves, rendait cette législation inapplicable. Ils ont sans doute raison $\left({ }^{59}\right)$. Il devait toutefois y avoir parfois une exception qu'illustrerait le papyrus oxyrhynchite.

J'ajouterai un document papyrologique au dossier. Un texte du $\mathrm{IV}^{\mathrm{e}}$ siècle montre que, si l'emploi du fouet est légalement permis pour châtier les esclaves, il n'est toutefois pas souhaitable. Il s'agit d'un édit du praeses de Thébaïde qui, de nouveau, refléterait la législation impériale, de Constantin, par exemple $\left({ }^{60}\right)$.

Conclusions. - Récapitulons par une réponse aux questions posées en début d'article : quel genre de méfaits ou de maltraitances les esclaves subissent-ils, de la part de qui, pour quelles raisons, obtiennent-ils réparation et comment, pourquoi et comment sont-ils punis par leur maître ?

Les sévices dont sont victimes les esclaves montrent une grande variété. Ils sont insultés $\left({ }^{61}\right)$, enfermés ou emprisonnés $\left({ }^{62}\right)$, enlevés et séquestrés $\left({ }^{63}\right)$, battus, torturés et brûlés $\left({ }^{64}\right)$, ils reçoivent des coups $\left({ }^{65}\right)$ entraînant des blessures $\left({ }^{66}\right)$ et même la mort $\left({ }^{67}\right)$, ils sont sexuellement violentés $\left({ }^{68}\right)$. Ils sont aussi victimes

(58) H. HeInEN, « Amtsärzliche Untersuchung » [n. 56], pp. 194-202. Citation p. 202.

(59) Dans son livre, Réalités [n. 1], M. Morabito relèvent les « infractions » à la législation sur la protection des esclaves. Par exemple, même en cas de meurtre avéré de l'esclave, « la protection des intérêts des maîtres reste un problème important » (p. 184).

(60) P.Oxy. IX, 1186 (Thébaïde, $\mathrm{IV}^{\mathrm{e}}$ s.). Voir n. 3.

(61) P.Oxy. VI, 903.

(62) P.Oxy. XIV, $1643 ;$ P.Oxy. VI, 903.

(63) P.Fam.Tebt. 37 ; P.Oxy. VIII, 1120.

(64) P.Oxy. XIV, 1643 ; P.Oxy. VI, 903. - Brûlé : SB XX, 14085, II, 11.

(65) P.Ryl. II, 144 ; P.Oxy. VI, 903.

(66) P.Oxy. XXXIII, 2672.

(67) SB XX, 14085, II, 11 ; P.Hamb. I, 10.

(68) SB XIV, 11904. 
d'abus de confiance et de vols $\left({ }^{69}\right)$. Pour être complet, on ajoutera à cette liste les blessures accidentelles $\left({ }^{70}\right)$.

Tous ces méfaits sont le fait du maître de l'esclave ou d'autres personnes, étrangères ou non à l'entourage de l'esclave. Les raisons de leurs agissements nous échappent le plus souvent. Sauf en cas de méfait commis par les esclaves, nous ne savons pas pour quelles raisons ceux-ci sont punis. Enfin, quand il y a demande de réparation soit par l'esclave lui-même, soit par l'intermédiaire de son maître, on ignore la suite donnée à la requête. Bref, la documentation papyrologique de l'Égypte romaine nous laisse un peu sur notre faim. Mais, malgré sa relative pauvreté, elle montre une condition servile peu enviable.

Jean A. STRAUS

(69) Abus de confiance : BGU IV, 1079. — Vols : P.Ryl. II, 144 ; P.Oxy. LVIII, 3916 ; P.Lond. II, 157 a, p. 255.

(70) P.Oslo III, 95 ; P.Oxy. L, 3555 ; P.Oxy. LXIII, 4370. 